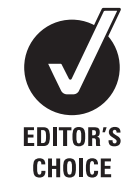

CHOICE

\title{
Elimination of central-venous-catheter- related bloodstream infections from the intensive care unit
}

\author{
Andrew G Longmate, ${ }^{1}$ Kirsteen S Ellis, ${ }^{1}$ Louise Boyle, ${ }^{1}$ Shaun Maher, ${ }^{1}$ \\ Chris J S Cairns, ${ }^{1}$ Suzanne M Lloyd, ${ }^{2}$ Colin Lang ${ }^{1}$
}

\begin{abstract}
- Additional appendices are published online only. To view these files please visit the journal online (http:// qualitysafety.bmj.com).

${ }^{1}$ Intensive Care Unit, Stirling Royal Infirmary, NHS Forth Valley, Stirling, UK

${ }^{2}$ Robertson Centre for Biostatistics, University of Glasgow, Glasgow, UK
\end{abstract}

Correspondence to Dr Andrew G Longmate, Intensive Care Unit, Stirling Royal Infirmary, NHS Forth Valley, Stirling FK8 2AU, UK; alongmate@nhs.net

Part of this work was previously reported as an abstract at the International Forum on Quality and Safety in Healthcare (Berlin, March 2009).

Accepted 3 July 2010
Introduction: Central-venous-catheter (CVC)-related bloodstream infection (CRBSI) is a complication of intensive care stay which can have important adverse consequences for both patient and institution. There are a number of evidence-based interventions that reduce CRBSI, but it is recognised that consistently applying the best evidence every time is a challenge.

Methods: The authors set out to reduce CRBSI and introduced interventions in our intensive care unit (ICU) over a 4-year period using a quality improvement approach. In a setting supportive to change and improvement, the authors established infection surveillance and introduced bundles of care processes relating to insertion and maintenance of CVCs. The changes were supported by educational interventions. The authors measured care processes and outcomes, and used statistical process control charts to illustrate changes. The final 18 months of the work was performed in the context of a national safety improvement programme (The Scottish Patient Safety Programme).

Results: Following interventions, the annual CRBSI rate fell from 3.4 to $0 / 1000$ patient days with zero episodes during the final 19 months of the study.

Conclusions: The authors describe a significant reduction in CRBSI for the first time in a UK ICU. The authors summarised and simplified what to do, measured and provided feedback on outcomes, and improved expectations of performance standards for care processes. The authors believe that these approaches are worthy of serious consideration elsewhere.

\section{INTRODUCTION}

Central-venous-catheter (CVC)-related bloodstream infections (CRBSIs) are an important cause of hospital-acquired infection associated with morbidity, mortality and cost. ${ }^{1-3}$ Several interventions prevent them, ${ }^{4}$ and a study in 103 intensive care units (ICUs) in Michigan $^{5}$ demonstrated a reduction from
7.7 to $1.4 / 1000$ catheter days following implementation of five care processes including hand hygiene, chlorhexidine skin antisepsis, barrier precautions during insertion, avoidance of femoral site and removal of unnecessary catheters. The Michigan Keystone interventions were introduced in the context of an organisational framework designed to support translation of evidence into practice. ${ }^{6}$ Some have called for widespread implementation and adoption of evidence-based guidelines ${ }^{7}$ and these five care processes $^{8}$ as part of a central line bundle. ${ }^{9}{ }^{10}$ Others have concluded that these practices might be worthy of wider implementation but that a further study would be helpful. ${ }^{11}$ Reduction of CRBSI has not been described in a UK ICU setting. We set out with this intention.

\section{METHODS}

Stirling Royal Infirmary is a general hospital serving 270000 people within Forth Valley. ICU CRBSI incidence was measured over a continuous 4-year period from 1 September 2005 to 31 August 009. Every patient admitted for more than $48 \mathrm{~h}$ in the adult medical and surgical nine bedded ICU who during part or whole of their admission had a CVC had a daily assessment until discharge for the occurrence of a CRBSI using Hospitals in Europe Links for Infection Control Surveillance (HELICS) ${ }^{12}$ dataset (online appendix 1) and definition Catheter Related Infection 3 (CRI 3) (online appendix 2). CVC was defined as any intravenous catheter ending at or near the heart. Flow charts to support definitions and diagnostic category were used (online appendices 3,4). A device day was defined by a patient having a single CVC for a whole or part $24 \mathrm{~h}$ period; two 
catheters for a part or whole $24 \mathrm{~h}$ period was defined as two device days and so on. The method of counting all catheter days as the denominator used by HELICS differs from that used by the National Nosocomial Infections Surveillance System ${ }^{13}$ and Centers for Disease Control and Prevention who use a single patient day as the denominator, even if the patient has two catheters on that given day. Infection incidence per 1000 device days and 1000 patient days was displayed on statistical process control (SPC) charts. ${ }^{14}{ }^{15}$ Confirmation that every patient was captured was made by crosschecking all names against the ICU admission book and the ICU 'Ward Watcher' database. CVCs removed had their tips routinely sent for modified Maki roll ${ }^{16}$ testing (online appendix 5).

Between 1 September 2005 and 31 December 2006, we focused on infection surveillance (ventilator associated pneumonia (VAP), methicillin-resistant Staphylococcus aureus and CRBSI) and performed interventions aimed at improving hand hygiene practices. The first year's CRBSI incidence was 3.4/1000 catheter days. We noted that CRBSI preventive practices at insertion including using chlorhexidine antisepsis and full aseptic technique were not consistently performed. For established CVCs, there was variation in hand-hygiene practices, techniques for commencing injections and infusions, and an inconsistent approach for removal. Common practice was to suggest routine replacement after 7 days sometimes using catheter exchange over a guide wire. These practices are not widely accepted as strategies to prevent CRBSI. There was no daily prompt to remove established CVCs. Although aware of these inconsistencies, we were unable to measures process reliability.

We formed a group consisting of three of our nine consultant clinicians, our infection surveillance nurse and two ICU charge nurses whose stated aim was to reduce CRBSIs in ICU. Two consultants had responsibility for training doctors in Anaesthesia and Intensive Care. Interventions were gradual and iterative commencing January 2007 and were supported by the Critical Care Development Group (our management forum). The ICU had a culture supporting improvements having previous success amalgamating two units into one and introducing renal replacement therapy. Initially, there was minimal hospital administrative role in the project, and although we had no prior experience with performance change (improvement programmes) there was palpable local ownership of the improvement efforts which were seen to come from within the ICU. Ethical approval was viewed as unnecessary, and confirmed by the local ethics committee, since there was no specific research question, and activities were viewed as good practice and routine in other centres. Interventions to reduce $\mathrm{VAP}^{17}$ ran from March 2007 to August 2009.

We agreed interventions derived from published strategies and developed insertion and maintenance bundles. Although these might appear slightly different from those described in Michigan, ${ }^{5}$ aseptic technique and maximal sterile barrier precautions rather than 'hand hygiene' were stipulated at the time of insertion, since this correlated with evidence. ${ }^{18}$ The daily prompt for catheter removal was included under maintenance, not insertion.

\section{Insertion bundle}

1. Use aseptic technique and maximal barrier precautions (cap and mask, surgical scrub, sterile gown and gloves).

2. Use $2 \%$ chlorhexidine $/ 70 \%$ alcohol solution skin antisepsis.

3. Avoid femoral site for insertion where possible (use the internal jugular or subclavian route).

4. Use CVC insertion checklist. This self-adhesive checklist highlighted the points above and other details including the operator name and whether they had completed the CVC education package. The 'checklist sticker' served as a record of the procedure in the clinical notes.

We stipulated that the operator and assistant should be trained (as defined by having performed the CVC insertion education package and having placed three supervised CVCs).

\section{Maintenance and removal}

- CVC removal as soon as not required using a daily removal prompt.

- Perform hand hygiene before handling CVC.

- Clean injection ports with alcohol wipe before use.

- Avoid use of three way taps where possible and use needle-less adaptors for injection ports.

- Perform daily dressing inspection and, if soiled, clean site with chlorhexidine and replace dressing.

- Use a dedicated lumen for total parenteral nutrition.

- We discouraged blood sampling from CVCs unless absolutely necessary.

- We discouraged catheter exchange over guide wire.

\section{BEHAVIOUR CHANGE INTERVENTIONS AND IMPLEMENTATION STRATEGIES}

Introduction of the bundles was supported by active engagement of staff, educational programmes, measurement and feedback of outcomes, organisational change and, later, the introduction of the Scottish Patient Safety Programme ${ }^{19}$ and measurement of insertion bundle processes (online appendix 6). 


\section{RESULTS}

Figure 1 shows all or none insertion bundle reliability over time annotated to show identification and resolution of causes of incomplete reliability. Detail is given in the online appendix 6. Reliability increased between March 2008 and August 2009.

Table 1 lists the patient characteristics comparing the baseline preintervention from the 1st year with that of the $3 \mathrm{rd}$ and 4 th years. Interventions commenced halfway through year 2 and continued iteratively until the end of year 4 .

Figures 2, 3 show monthly and quarterly SPC charts demonstrating a reduction in infections over time. There is an association with the fall in incidence and the start of interventions and commencement of process measurement.

Table 2 lists the infections, patients, patient days and infection incidence per annum from 2005 to 2009. The incidence ratio was calculated as postintervention year rate/year 1 rate; exact CIs and p values calculated using Stata v 10.0. CRBSI incidence fell from 3.38 to $0.46 / 1000$ device days between year 1 and 3 with an incidence rate ratio of 0.137 (95\% CI 0.003 to 0.990 ) representing a significant $86.3 \%$ reduction $(\mathrm{p}=0.0134)$. Between year 1 and 4 , the incidence fell from 3.38 to $0 / 1000$ device days with an incidence rate ratio of $0(95 \%$ CI 0.0 to $0.63)$ representing a significant reduction $(p=0.0025)$.

\section{DISCUSSION}

The understanding of how to change behaviours and reliably deliver evidence-based interventions is evolving. Although impossible to establish causality, we noted a close association between reliable implementation of interventions known to prevent CRBSI and a fall in the annual CRBSI incidence from 3.38 to $0 / 1000$ device

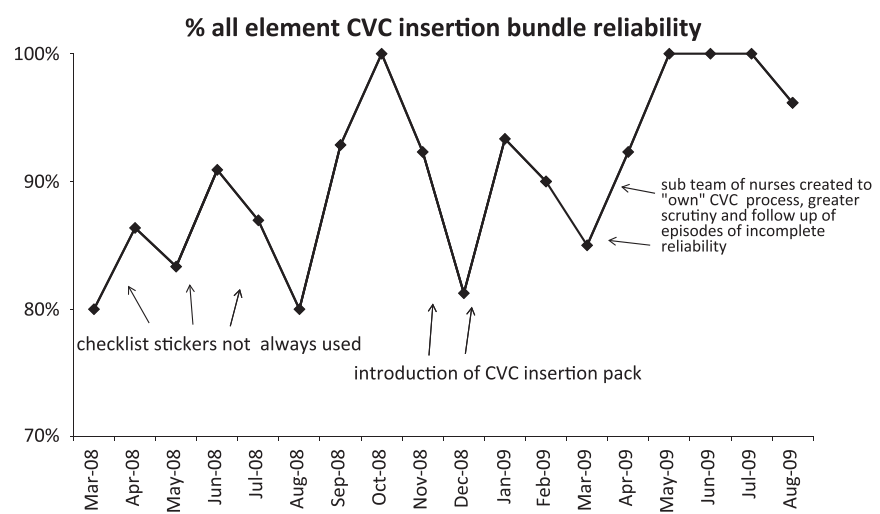

Figure 1 All or none insertion bundle reliability over time annotated to show identification and resolution of causes of incomplete reliability. Detail is given in the online appendix 6 . Reliability increased between March 2008 and August 2009. days. High insertion bundle reliability $(>80 \%)$ was demonstrated over a continuous and prolonged period (18 months) and associated with zero infections. Incomplete reliability was due to failure to place a checklist in the notes, presumably a less important intervention than the other insertion bundle elements. Changes and interventions were iterative and in the context of the social conditions of our ICU overlain latterly by the Scottish Patient Safety Programme ${ }^{19}$; a national breakthrough collaborative ${ }^{20}$ including national learning meetings, leadership engagement, access to content and improvement experts, support with collection and interpretation of data and the goal of reducing CRBSI. There are similarities to the Michigan Keystone project ${ }^{5}{ }^{6}$ which included designation of clinical and nurse leaders, teams receiving supporting information on the efficacy of components of the intervention, suggestions for implementation and instruction on methods of data collection, training in safety, regular conference calls, coaching and twiceyearly statewide meetings. Key components have been summarised as a focus on systems, engagement of local interdisciplinary teams to assume ownership, centralised support for technical work, encouragement of local adaption of intervention and the creation of a collaborative culture in the local unit and larger system. ${ }^{6}$ In this sense, it is important to acknowledge that the outcome changes we witnessed are likely to be more complex than the simple introduction of a checklist or bundles and probably reflect more complex social and cultural behaviour changes. ${ }^{21}$ The observed reduction in CRBSI could be seen as a surrogate and indirect measure of staff performance change. ${ }^{22}$ Further study and understanding of the relationship between social behaviourchange interventions and staff behaviours is required. It is not clear how long this kind of improvement can be sustained, and what the key elements will be to maintaining it.

As with the Michigan ${ }^{5}$ study, data collection and interventions were non-blinded using non-controlled before and after cohorts. Insertion processes were measured only for ICU-placed CVCs. We did not measure maintenance processes; nor did we perform a health economic assessment, although others have demonstrated significant potential cost savings from preventing infection. ${ }^{23-25}$ We made no formal assessment of potential adverse effects of the intervention, although none were brought to our attention. Although we described educational interventions, we did not objectively quantify the dose or staff knowledge before or after.

Regression to the mean is an unlikely explanation for improvement, since the baseline infection rate was similar to the rates reported from other Scottish ICUs 


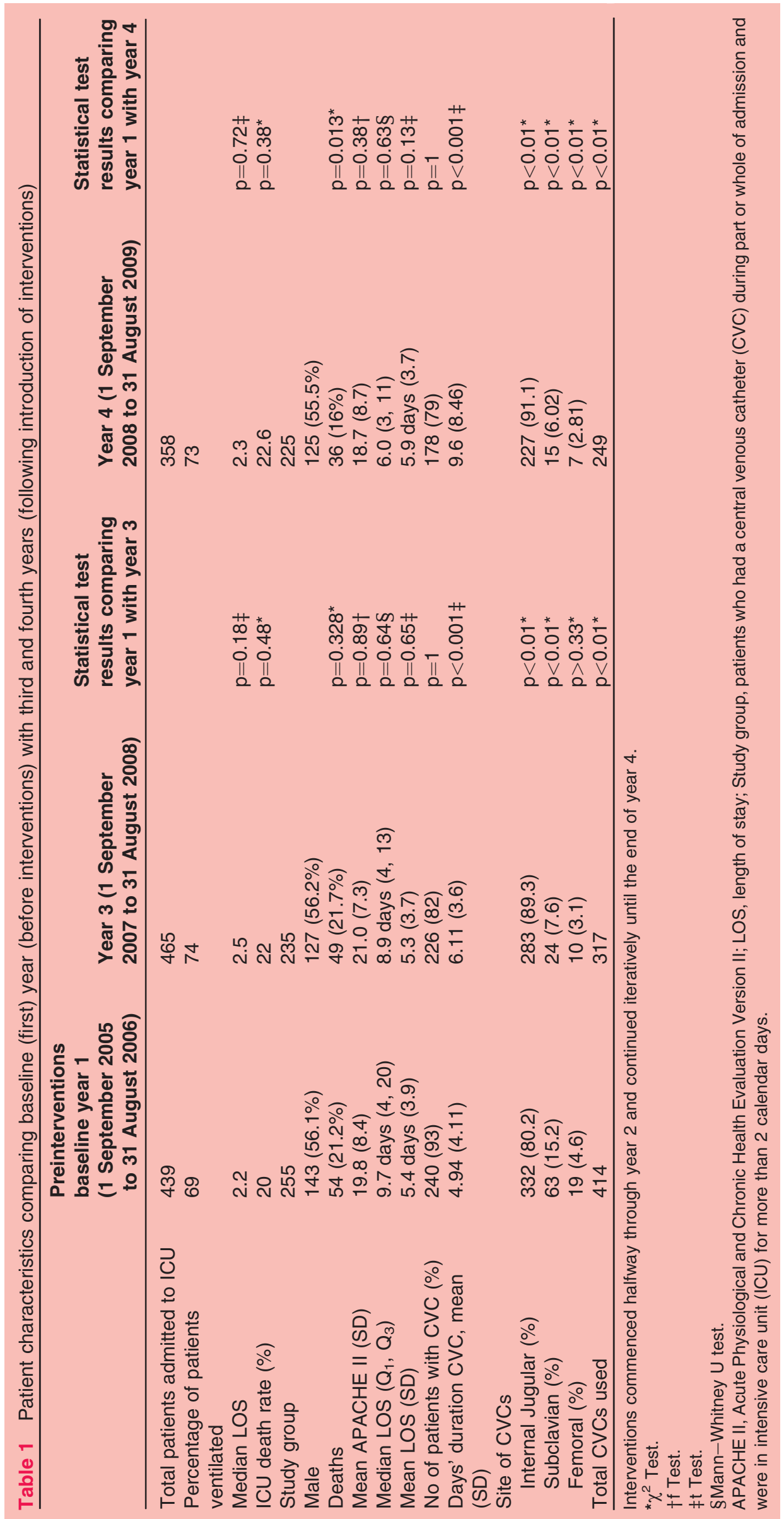


Figure 2 U chart. Monthly central-venous-catheter-related bloodstream infection (CRBSI) acquisition as rate per device day (number of infections divided by the device days/month). The plot demonstrates the common cause variation before the interventions start. Special cause variation (downwards shift) is evidenced by a run of $>6$ points below the centre line from February 2008.

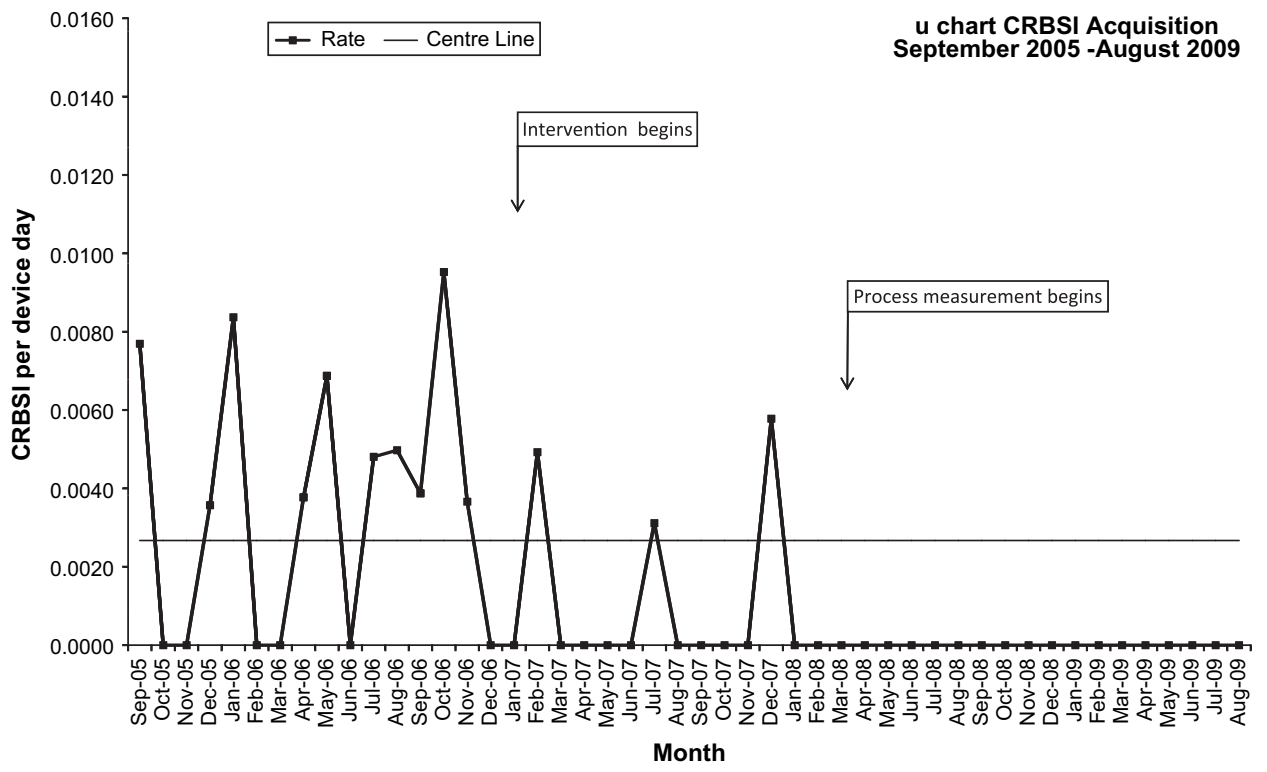

(4.2/1000 device days). ${ }^{26}$ The SPC chart demonstrated a common cause variation in the baseline period, further supporting the likelihood that the change to zero infection rate represented special cause variation.

The following discussion concerns results shown in table 1. There is no difference in mean Acute Physiological and Chronic Health Evaluation Version II score from year to year. However, there were some important differences. The total number of CVCs used fell significantly from 414 in year 1 to 317 in year 3 and 249 in year 4. There is a non-significant reduction in the percentage of patients per year with a CVC from 93\% (baseline) to $79 \%$ (year 4). There was a higher mean duration of CVC placement postinterventions, increasing significantly from 4.9 days (baseline) to 6.1 and 9.6 days in years 3 and 4 respectively. This might reflect a cohort of patients who previously had a CVC for a short duration of time but who are now managed without. Fewer CVCs would predispose to reduced infection incidence, but longer catheter placement durations would predispose to increased incidence. Internal jugular catheters increased significantly from $80.2 \%$ (baseline) to $89.3 \%$ (year 3) and $91.1 \%$ (year 4 ). Femoral catheters fell significantly from $4.6 \%$ (baseline) to $2.8 \%$ (year 4). Subclavian catheters fell significantly from $15.2 \%$ (baseline) to $6.0 \%$ (year 4). The increased internal jugular and decreased
Figure 3 Quarterly centralvenous-catheter-related bloodstream infection (CRBSI) acquisition as rate per 1000 device days and per 1000 patient days (number of infections divided by the device days and patient days respectively $\times 1000$ per month). CDC, Centers for Disease Control and Prevention; HELICS, Hospitals in Europe Links for Infection Control Surveillance.
Quarterly u chart: CRBSI/1000 device days (HELICS) and /1000 patient days (CDC)

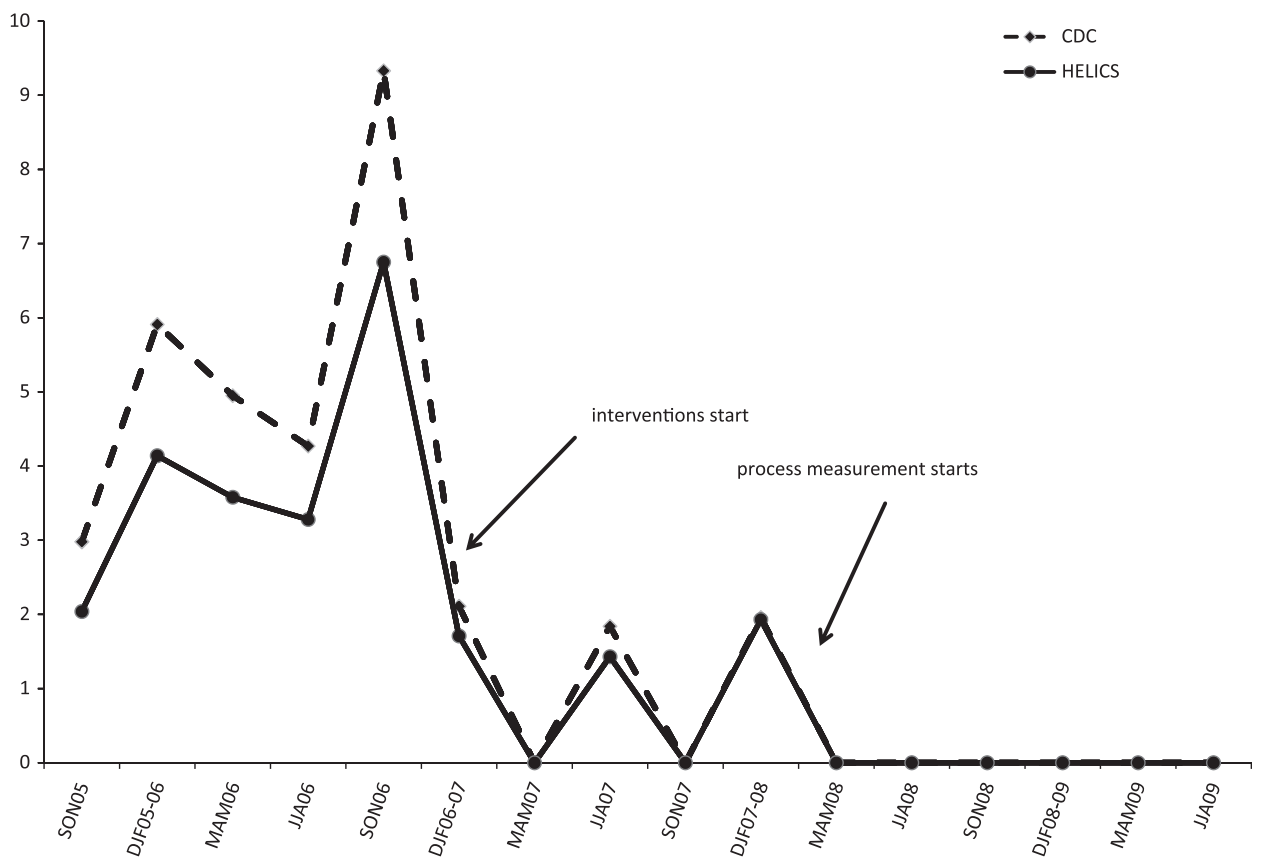




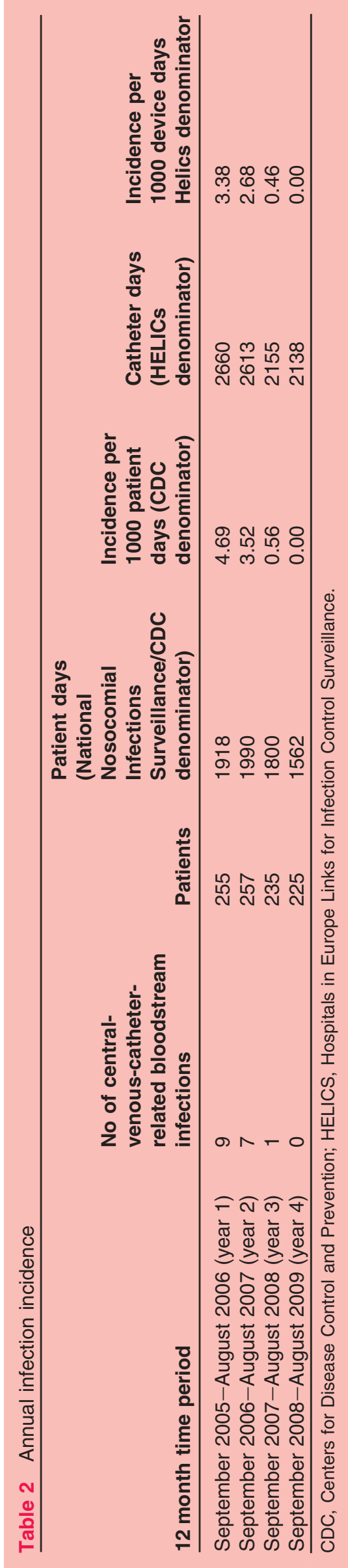

femoral site is consistent with the insertion bundle, but it is unclear why there was a reduction in subclavian catheters. There was a non-significant reduction in median length of stay from 9.7 to 6.0 days. Unadjusted mortality fell from $21.2 \%$ (baseline) to $16 \%$ (year 4) in patients with CVCs. Multiple factors influence mortality.

\section{CONCLUSION}

We describe a significant reduction in CRBSI (with zero episodes in the final 19 months of study) in a UK ICU. We summarised and simplified what to do, measured and provided feedback on outcomes, and improved the culture by building expectations of performance standards for work processes. ${ }^{21}$

Acknowledgements We would like to thank M Williamson, $Y$ Curran and Health Protection Scotland, A Duff, M Mallice, L Shepherd, P Scott, M Fraser, L Hutchison, J Grant and the ICU staff. We would also like to thank R Lloyd and the anonymous reviewers for their comments on earlier versions of this manuscript.

Funding Health Protection Scotland provided funding for a nurse salary for the second year of the project. Health Protection Scotland, Clifton House, 1-7 Clifton Place, Glasgow G3 7LN.

Competing interests AGL has recently completed a Scottish Patient Safety Programme Fellowship. SM is currently undertaking the same. LB is Nursing Lead for the Critical Care work stream of the Scottish Patient Safety Programme at Stirling Royal Infirmary.

Provenance and peer review Not commissioned; externally peer reviewed

\section{REFERENCES}

1. Mermel LA. Prevention of intravascular catheter-related infections. Ann Intern Med 2000;132:391-402.

2. Burke JP. Infection control-a problem for patient safety. N Engl J Med 2003;348:651-6.

3. Soufir L, Timsit JF, Mahe C, et al. Attributable morbidity and mortality of catheter-related septicemia in critically ill patients: a matched, risk adjusted cohort study. Infect Control Hosp Epidemiol 1999;20:396-401.

4. O'Grady NP, Alexander M, Dellinger P, et al. Guidelines for the prevention of intravascular catheter-related infections. Infect Control Hosp Epidemiol 2002;23:759-69.

5. Pronovost $\mathrm{P}$, Needham D, Berenholtz $\mathrm{S}$, et al. An intervention to decrease catheter-related bloodstream infections in the ICU. N Engl J Med 2006;355:2725-32.

6. Pronovost P, Berenholtz S, Needham D. Translating evidence into practice: a model for large scale knowledge translation. BMJ 2008;337:963-5.

7. O'Grady NP, Gerberding JL, Weinstein RA, et al. Patient safety and the science of prevention: The time for implementing the guidelines for the prevention of intravascular catheter-related infections is now. Crit Care Med 2003;31:291-2.

8. Wenzel RP, Edmond MB. Team based prevention of catheter-related infections. N Engl J Med 2006;355:2781-3.

9. Institute for Healthcare Improvement. Implement the Central Line Bundle. http://www.ihi.org/lHI/Topics/CriticalCare/IntensiveCare/ Changes/ImplementtheCentralLineBundle.htm (accessed 8 Mar 2009).

10. Central Line Insertion Bundle. Scottish Intensive Care Society Audit Group. 2008. http://www.sicsag.scot.nhs.uk/SubGroup/VAP-C-lineBundle-080123.pdf (accessed 24 Mar 2009).

11. Ranji SR, Shetty K, Posley KA, et al. Prevention of Health Care Associated Infections. In: Shojania KG, McDonald KM, Wachter RM et al, eds. Closing the Quality Gap: A Critical Analysis of Quality Improvement Strategies. Technical Review 9 (prepared by Stanford University-UCSF Evidence-based Practice Center under contract No 290-02-0017). Vol. 6. AHRQ Publication No 04(07)-0051-6. Rockville MD: Agency for Healthcare Research and Quality. 2007. http://www. 
ahrq.gov/downloads/pub/evidence/pdf/qualgap6/hainfgap.pdf (accessed 29 Jan 2008).

12. Hospital In Europe Link for Infection Control through Surveillance (HELICS). Surveillance of Nosocomial Infections in Intensive Care Units (2004) Protocol Version 6.1. Project commissioned by the EC/ DG SANCO/F/4. Agreement Reference number: VS/1999/5235 (99CVF4-0125). http://www.helics.univ-lyon1.fr/helicshome.htm (accessed 28 Jan 2008)

13. National Nosocomial Infections Surveillance System. National Nosocomial Infections Surveillance (NNIS) System Report, data summary from January 1992 through June 2004, issued October 2004. Am J Infect Control 2004;32:470-85.

14. Benneyan JC, Lloyd RC, Plsek PE. Statistical process control as a tool for research and healthcare improvement. Qual Saf Health Care 2003:12:458-64.

15. Benneyan JC. Statistical quality control methods in infection control and hospital epidemiology, part ii: chart use, statistical properties, and research issues. Infect Control Hosp Epidemiol 1998;19:265-83.

16. Maki DG, Weise CE, Sarafin HW. A semiquantitative culture method for identifying intravenous catheter related infections. N Engl J Med 1977;296:1305-9.

17. Hawe C, Ellis KS, Cairns CJS, et al. Reduction of ventilator associated pneumonia: active versus passive guideline implementation. Intensive Care Med 2009;35:1180-6.

18. Raad II, Hohn DC, Gilbreath BJ, et al. Prevention of central venous catheter-related infections by using maximal sterile barrier precautions during insertion. Infect Control Hosp Epidemiol 1994; 15:231-8

19. Scottish Patient Safety Alliance. Scottish Patient Safety Programme. http://www.patientsafetyalliance.scot.nhs.uk/programme (accessed 10 Jun 2009).

20. Break through series: IHIs collaborative model for achieving breakthrough improvement. http://www.ihi.org/lHI/Results/WhitePapers/

TheBreakthroughSeriesIHIsCollaborativeModelforAchieving +Breakthroughlmprovement.htm (accessed 25 May 2010).

21. Bosk CL, Dixon-Woods M, Goeschel CA, et al. Reality check for checklists. Lancet 2009;374:444-5.

22. Davidoff F. Heterogeneity is not always noise. Lessons from improvement. JAMA 2009;302:2580-6.

23. Eggimann $\mathrm{P}$, Harbarth $\mathrm{S}$, Constantin $\mathrm{MN}$, et al. Impact of a prevention strategy targeted at vascular-access care on incidence of infections acquired in intensive care. Lancet 2000;355:1864-8.

24. Coopersmith CM, Redmann TL, Zack JE, et al. Effect of an education programme on decreasing catheter related bloodstream infections in the surgical intensive care unit. Crit Care Med 2002;30:59-64.

25. Berenholtz SM, Pronovost PJ, Lipsett PA, et al. Eliminating catheterrelated blood stream infections in the intensive care unit. Crit Care Med 2004;32:2014-20.

26. Baseline ITU infections. http://www.documents.hps.scot.nhs.uk/hai/ sshaip/publications/icu-surveillance/icu-surveillance-report.pdf. 